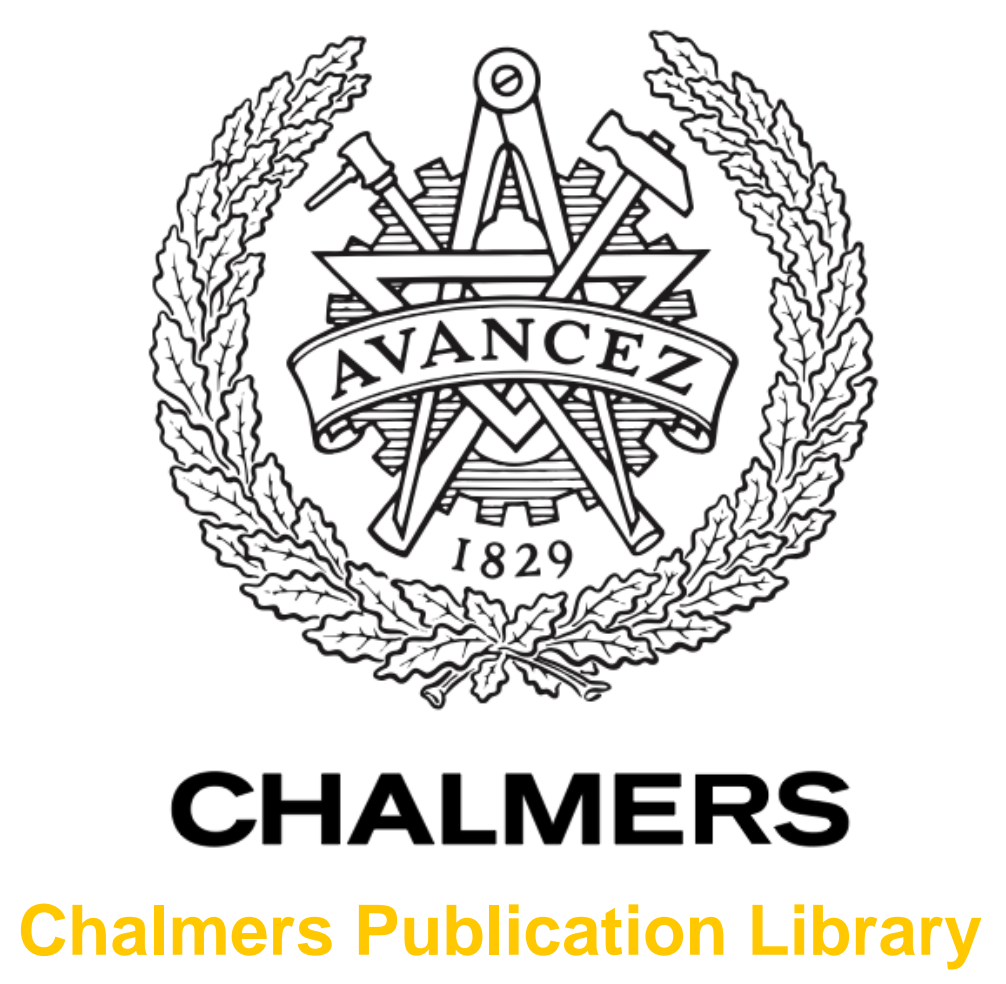

The flow around a simplified tractor-trailer model studied by large eddy simulation

This document has been downloaded from Chalmers Publication Library (CPL). It is the author's version of a work that was accepted for publication in:

Journal of Wind Engineering and Industrial Aerodynamics (ISSN: 0167-6105)

Citation for the published paper:

Östh, J. ; Krajnovic, S. (2012) "The flow around a simplified tractor-trailer model studied by large eddy simulation". Journal of Wind Engineering and Industrial Aerodynamics, vol. 102 pp. 36-47.

http://dx.doi.org/10.1016/j.jweia.2011.12.007

Downloaded from: http://publications.lib.chalmers.se/publication/154588

Notice: Changes introduced as a result of publishing processes such as copy-editing and formatting may not be reflected in this document. For a definitive version of this work, please refer to the published source. Please note that access to the published version might require a subscription. 


\title{
The Flow around a simplified tractor-trailer model studied by large eddy simulation
}

\author{
Jan Östh*, and Siniša Krajnović \\ Division of Fluid Dynamics, \\ Department of Applied Mechanics, \\ Chalmers University Of Technology, \\ SE-412 96 Göteborg, Sweden \\ www.tfd.chalmers.se/ sinisa
}

\begin{abstract}
Large-eddy simulation (LES) is used to study the flow around a simplified tractor-trailer model. The model consists of two boxes placed in tandem. The front box represents the cab of a tractor-trailer road vehicle and the rear box represents the trailer. The LES was made at the Reynolds number of $0.51 \times 10^{6}$ based on the height of the rear box and the inlet air velocity. Two variants of the model were studied, one where the leading edges on the front box are sharp and one where the edges are rounded. One small and one large gap width between the two boxes were studied for both variants. Two computational grids were used in the LES simulations and a comparison was made with available experimental force measurements. The results of the LES simulations were used to analyze the flow field around the cab and in the gap between the two boxes of the tractor-trailer model. Large vortical structures around the front box and in the gap were identified. The flow field analysis showed how these large vortical structures are responsible for the difference in the drag force for the model that arises when the leading edges on the front box are rounded and the gap width is varied.
\end{abstract}

Keywords: Vehicle Aerodynamics, Large Eddy Simulation, Simplified Tractor-trailer, Gap Flow, CFD, Bluff Body Aerodynamics, Tandem Configuration

\section{Introduction}

The aerodynamic performance of heavy vehicles is an area in which improvements can be made even though there have been great achievements in the past half century. The aerodynamic forces that act on a vehicle during driving affect the operation of the vehicle in different ways. The side and lift forces may cause instabilities and handling problems. The drag force affects the velocity and acceleration of the vehicle as well as the fuel consumption. For commercial vehicles, overcoming the drag requires a lesser part of the total fuel, that

*Email address: ojan@chalmers.se (Jan Östh)

Preprint submitted to Journal of Wind Eng. Ind. Aerodyn.

January 17, 2012 
goes to overcome all the driving resistances (such as drag, rolling and climbing resistance) during cruising, when compared with a passenger car. The energy that can be saved for commercial vehicles by decreasing the aerodynamic drag is still apparent.

The pressure drag, which makes up the major part of the aerodynamic drag on a tractortrailer unit, essentially comes from four parts of the vehicle: the forebody of the tractor, the rear part of the trailer, the underhood plus wheels and the gap between the tractor and the trailer. The latter is a large contributor to drag. The shape of the tractor cab will influence what the flow conditions will be for the trailer. The contribution to the overall drag coefficient of a tractor-trailer unit from the gap is influenced by the width of the gap between the tractor cab and trailer. The gap width will henceforth be denoted by $g$ and expressed in non-dimensional form $g / b$ or sometimes $g / \sqrt{A}$, where $b$ and $A$ are characteristic length and area, respectively, of the specific model studied. In general, the smaller the gap is, the smaller will the positive contribution to the drag coefficient of the gap be. This is of course also dependent on the relationship between the heights of the tractor and the trailer. The drag coefficient of simplified tractor-trailer units with a cab with rounded shape is smallest for zero gap widths and increases slightly with increasing gap width. Around a gap width of $g / \sqrt{A} \approx 0.5$ ( $A$ is the model cross-sectional area) a sudden increase in drag occurs (McCallen et al. [2000], Allan [1981], Hammache and Browand [2002]). This increase in drag is completely due to an increase in the drag of the trailer alone. The drag of the tractor cab varies very little with increasing gap widths (Allan [1981], Hammache and Browand [2002]). As noted above, the drag of the entire unit depends a great deal on the flow in the gap, and even more so when the vehicle is operating in cross-wind environments. With an increasing yaw angle of the oncoming airflow, the drag for the unit is significantly increased and units with sharp-edged cabs are affected most (Hucho [1998], Barnard [1996]). One reason for this is that the region of stagnation on the trailer increases when the air flow comes from the side. Various successful add-on devices for reducing the contribution to the overall drag from the gap are today standard practice among heavy vehicle manufacturers. These are, e.g., deflector plates mounted on the cab roof and cab side extenders that direct the air over the trailer and fairings mounted on the trailer, which help flow attachment in the gap during cross-wind conditions. Other devices such as gap seals have proven to be able to further reduce the drag but are not yet in common use. For a historical view of the development of truck aerodynamics see Cooper [2002, 2003].

Allan [1981] made aerodynamic measurements in the wind tunnel of Soupthampton on a simplified tractor-trailer model at Reynolds number $R e_{b}=0.51 \times 10^{6}$ based on the height of the trailer box, $b$. The model he used is also the one used in the present numerical study, see Fig. 1. The model consists of one front box representing the tractor cab and a rear box representing the trailer. The front box has a height, width and depth of $0.92 b, 0.92 b$ and $0.67 b$, respectively. The rear box has a height, width and depth of $b, b$ and $2.5 b$, respectively. The ground clearance of the front and rear box is $0.21 b$ and $0.5 b$, respectively. This setup gives a realistic placement of the cab and trailer with respect to the standard shape of European tractor-trailer trucks at the time. Two variants of the model were investigated by Allan [1981], one model with sharp leading edges on the front box and another with rounded edges. The width of the gap between the two boxes was varied and the drag was 
measured for $0^{\circ}$ yaw angle. The results are shown in Fig 2. It was found that the value of the drag coefficient of the model with sharp leading edges on the front box was more stable with respect to increased gap width. The model with rounded edges exhibited significantly smaller drag coefficient for small gap widths but the drag increased dramatically for larger gap widths and even gave a larger drag coefficient than for the sharp model. This behavior of the drag coefficient of another simplified tractor-trailer model with a rounded front was also found later in other experimental studies (Hammache and Browand [2002], Arcas et al. [2004]).

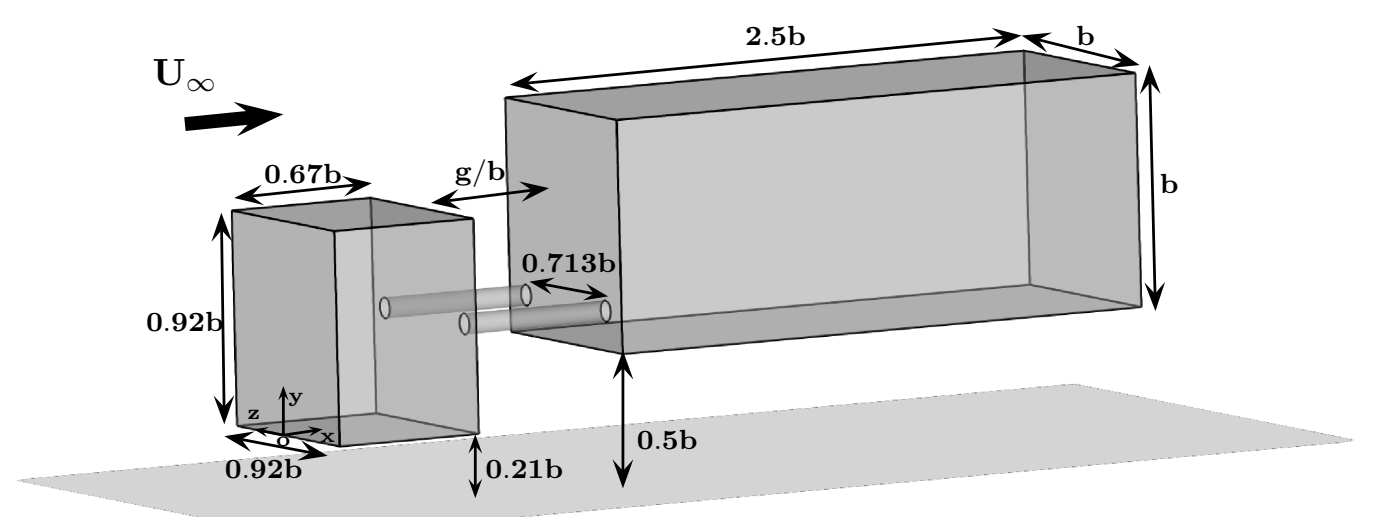

Figure 1: Dimensions of the model used in the experiments by Allan [1981] and in the present LES simulations. The variant with sharp leading edges on the front box is shown. 


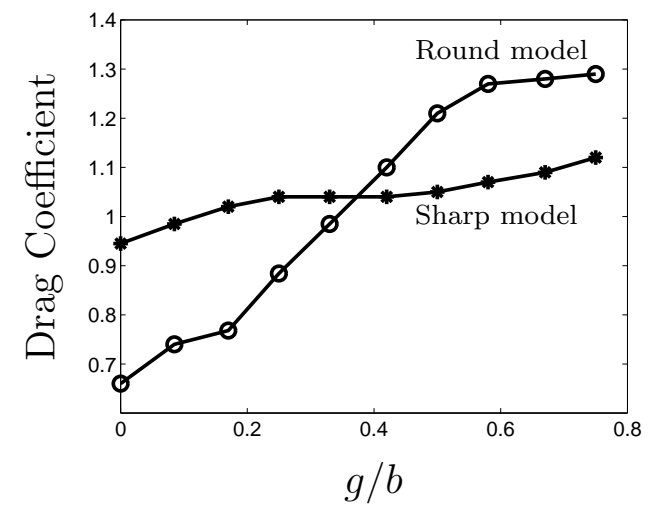

Figure 2: Experimental results for the drag coefficient for the two models investigated, redrawn from Allan [1981].

Large Eddy Simulations (LES) were used by Krajnović et. al. for a number of different bluff body flows, such as the flow around a generic train (Hemida et al. [2005]), a simplified bus (Krajnović and Davidson [2003]), a finite tall cylinder (Krajnović [2011]), and the ground vehicle Ahmed body reference model (Krajnović and Davidson [2005a,b]). See Krajnović [2009] for a further review of LES for bluff body flows. These studies have shown that LES provides useful information about the flow structures around vehicle-shaped bluff bodies at moderate Reynolds numbers and can correctly predict the global quantities, such as the drag coefficient. In LES the large scale turbulent motions are resolved directly while the influence of the smaller scales on the large scales is modelled (Pope [2000]). It is a transient method that enables the study of the large scale unsteady structures that are characteristic of bluff body flows.

The aim of the work in this paper is to explain the underlying flow physics of the disparities in the behavior of the drag coefficients of the two models in the study by Allan [1981] (see Fig. 2). LES should be well suited to simulate the massively separated flow around the simplified tractor-trailer model. This introductory part of the paper is followed by a section where the simplified tractor-trailer models used in the simulations are presented. Then follows a section that describes the numerical details used in the simulations which is followed by a section where results are presented and interpreted. In the last section the paper is summarized.

\section{Method}

\subsection{Description of the model}

In the present work, LES simulations of the flow around the tractor-trailer model from Allan [1981] are conducted. At a zero yaw angle, the flow physics will be explored around the model with square leading edges on the front box and the model with rounded leading edges on the front box. The general dimensions of the model are shown in Fig. 1. In the round model, both the horizontal and vertical leading edges on the front box are rounded 
with the non-dimensional radius $0.08 b$ which is the same as in the experiments by Allan [1981]. This necessarily extends the length of the front box for the round model by $0.08 b$. The two boxes are connected to each other with two cylinders of diameter $0.08 b$, which are placed horizontally in the gap at half of the height of the front box. The complete model was suspended from the arms of an overhead balance in the experiments. No locations or details of these arms were reported, and the arms are therefore neglected in the present simulations. Two different gap widths are investigated for both models, one small gap width of $g / b=0.17$ ( $\mathrm{b}=0.305 \mathrm{~m}$ is the width of the rear box) and one large gap width of $g / b=0.67$. The experimental results from Allan [1981] indicate that the sharp model has a drag coefficient of 1.02 and the round model 0.77 for $g / b=0.17$, based on the cross-sectional area of the rear box $\left(b^{2}=0.093 m^{2}\right)$. For $g / b=0.67$, the sharp model has a drag coefficient of 1.09 and the round model 1.28. The Reynolds number based on $b$ and the free-stream velocity in the experiments was $R e_{b}=0.51 \times 10^{6}$ and this is the same in the present simulations.

\subsection{Numerical set-up}

\subsubsection{LES Governing Equations}

The governing LES equations are the incompressible Navier-Stokes and the continuity equations filtered with the implicit spatial filter of characteristic width $\Delta$ :

$$
\frac{\partial \bar{u}_{i}}{\partial t}+\frac{\partial}{\partial x_{j}}\left(\bar{u}_{i} \bar{u}_{j}\right)=-\frac{1}{\rho} \frac{\partial \bar{p}}{\partial x_{i}}+\nu \frac{\partial^{2} \bar{u}_{i}}{\partial x_{j} \partial x_{j}}-\frac{\partial \tau_{i j}}{\partial x_{j}}
$$

and

$$
\frac{\partial \bar{u}_{i}}{\partial x_{i}}=0
$$

Here, $\bar{u}_{i}$ and $\bar{p}_{i}$ are the resolved velocity and pressure, respectively, and the bar over the variable denotes filtering.

The influence of the small scales of the turbulence on the large energy carrying scales in Eq. (1) appears in the SGS stress tensor, $\tau_{i j}=\overline{u_{i} u_{j}}-\bar{u}_{i} \bar{u}_{j}$. The algebraic eddy viscosity model originally proposed by Smagorinsky [1963] is used in the present work for its simplicity and low computational cost. The Smagorinsky model represents the anisotropic part of the SGS stress tensor, $\tau_{i j}$, as:

$$
\tau_{i j}-\frac{1}{3} \delta_{i j} \tau_{k k}=-2 \nu_{s g s} \bar{S}_{i j}
$$

where $\nu_{\text {sgs }}=\left(C_{s} f \Delta\right)^{2}|\bar{S}|$ is the SGS viscosity and

$$
\bar{S}_{i j}=\frac{1}{2}\left(\frac{\partial \bar{u}_{i}}{\partial x_{j}}+\frac{\partial \bar{u}_{j}}{\partial x_{i}}\right)
$$

is the resolved rate-of-strain tensor and $|\bar{S}|=\left(2 \bar{S}_{i j} \bar{S}_{i j}\right)^{\frac{1}{2}}$. The value of the Smagorinsky constant $C_{S}=0.1$ previously used for similar types of vehicle bluff body flows (Krajnović [2009]) is used in the present work. $f$ in the expression for the SGS viscosity is the van Driest damping function 


$$
f=1-\exp \left(\frac{n^{+}}{25}\right)
$$

where $n^{+}$is the wall normal distance in viscous units. The filter width, $\Delta$, is defined in this work as $\Delta=\left(\Delta_{1} \Delta_{2} \Delta_{3}\right)$, where $\Delta_{i}$ are the computational cell sizes in the three coordinate directions.

\subsubsection{Numerical method}

Equations (1) and (2) are discretized using a commercial finite volume solver, AVL Fire v2009.1 (AVL [2009]), for solving the incompressible Navier-Stokes equations using a collocated grid arrangement. The convective fluxes are approximated by a blend of $95 \%$ linear interpolation of second order accuracy (central differencing scheme) and of 5\% upwind differences of first order accuracy (upwind scheme). The diffusive terms containing viscous plus sub-grid terms are approximated by a central differencing interpolation of second order accuracy. It should be noted that for complex geometries using non-equidistant computational grids a commutation error is introduced since the formulation of Eq. (1) assumes that the filtering operation and spatial derivation commutes, which is not true on non-equidistant grids. This error is of the same order as the truncation error of the second order linear interpolation scheme (see e.g. Ghosal and Moin [1995]). The time marching procedure is done using the implicit second-order accurate three-time level scheme

$$
\left(\frac{d \phi}{d t}\right)_{n}=\frac{3 \phi^{n}-4 \phi^{n-1}+\phi^{n-2}}{2 \Delta t_{n}}, \Delta t_{n}=t-t_{n-1}=t_{n-1}-t_{n-2}
$$

where indices "n" and "n-1" denote the new and old time levels, respectively. To determine the pressure, the discrete form of the filtered continuity equation (Eq. 2) is converted into an equation for the pressure correction. The SIMPLE algorithm (Patankar and Spalding [1972]) is used to update the pressure and velocity fields so that they satisfy the continuity equation.

\subsubsection{Computational grids}

For all of the four cases, two different computational grids were used to check grid dependence of the results. The structured grids were made with the commercial grid generator software Ansys ICEM-CFD and consist of only hexahedral elements. The coarse grids consisted of 10.2, 10.3, 11.0 and 12.1 million cells for the square model with a small gap width (Case 1), the rounded model with a small gap width (Case 2), the square model with a large gap width (Case 3) and the rounded model with large gap width (Case 4), respectively. A horizontal cut in the grid for Case 2 is shown in Fig. 3. The finer grids consisted of 13.8, 14.6, 16.3 and 17.1 for Case 1-4, respectively. The grids consist of rectangular blocks, and the model enclosed in an O-grid. About $70 \%$ of all the cells are in the O-grid, which forms a belt with a width of $0.25 \mathrm{~b}$ around the front box and $0.4 \mathrm{~b}$ around the rear box. 


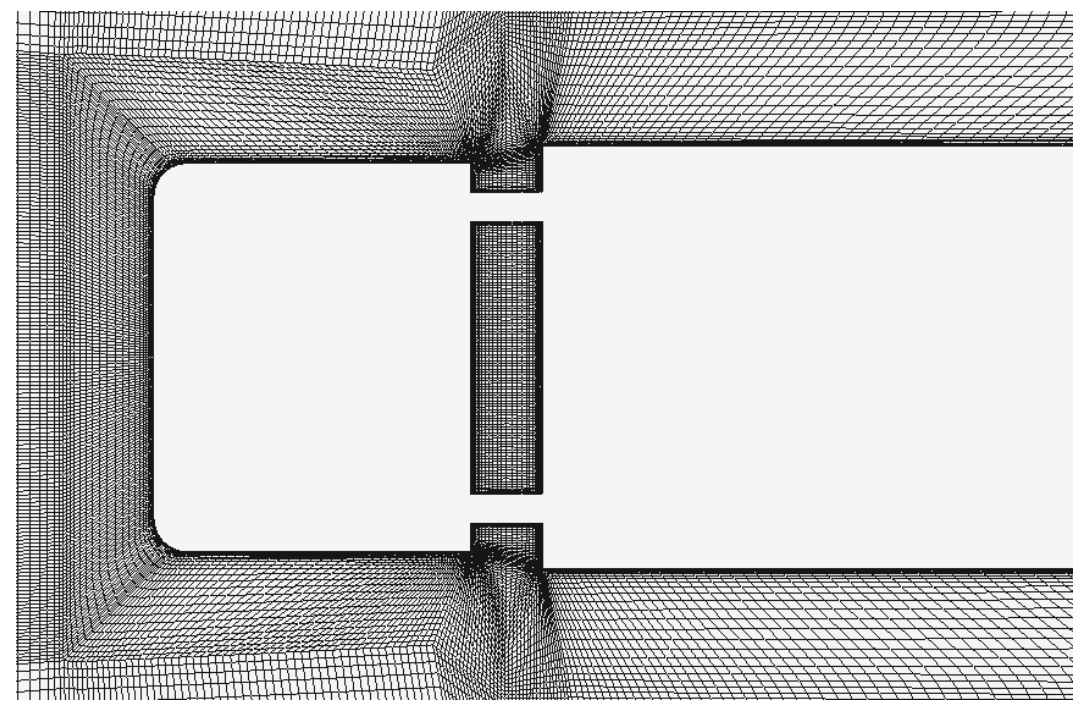

Figure 3: A horizontal cut in the coarse computational grid for Case 2. 


\subsubsection{Boundary conditions}

The computational domain is shown in Fig. 4. The size of the cross section corresponds to the wind tunnel used in the experiments by Allan [1981]. A uniform free-stream velocity of $U_{\infty}=24.4 \mathrm{~m} / \mathrm{s}$ is set at the inlet, which is the same as used in the wind tunnels tests. No value of the turbulence intensity of the free-stream velocity in the wind tunnel was reported by Allan [1981]. Thus, the steady velocity profile was used at the inlet in the present work. On the floor, the moving ground used in the experiments is simulated by setting the velocity component in the streamwise direction on the ground equal to $U_{\infty}$ together with the no-slip boundary condition. Since the velocity of the moving ground is equal to the free-stream velocity, there is no boundary layer on the floor and it consequently doesn't need to be resolved. The no-slip condition is used on the lateral walls and roof. However, the boundary layers are not resolved here. On the tractor-trailer model the no-slip condition is used. The homogeneous Neumann boundary condition is applied at the outlet. 


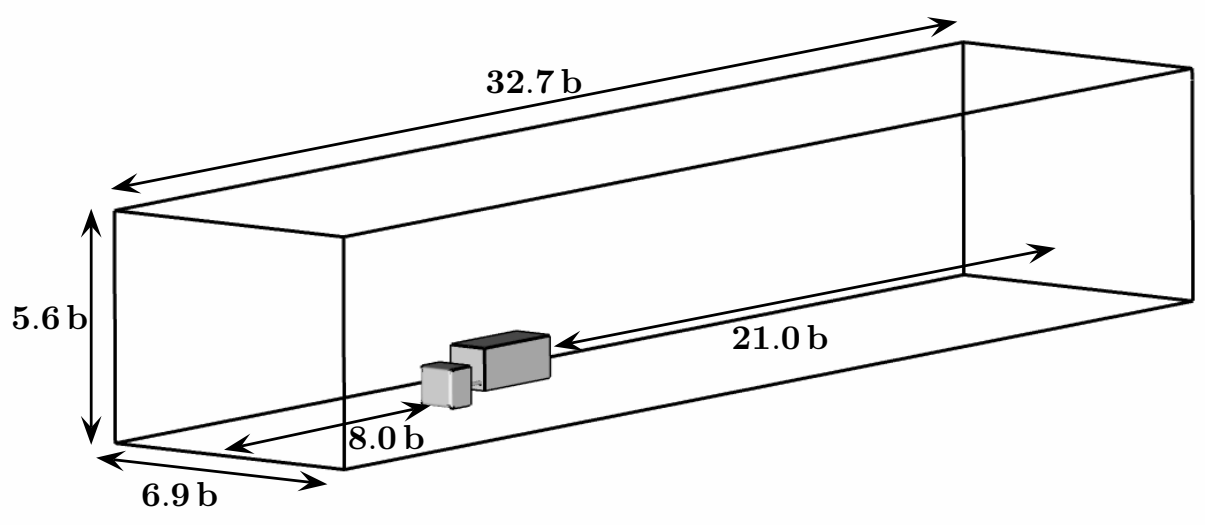

Figure 4: The computational domain used in the present LES simulations. $\mathrm{b}=0.305 \mathrm{~m}$.

\subsubsection{Spatial and temporal resolution}

For both the coarse and fine grids the wall normal resolution on the truck model is $n^{+}<1$ in all four cases. To achieve this, the size in normal direction of the first cell is $0.00016 b$. The maximum values of $n^{+}$are found on the very edges of the square geometries. For the coarse grids, the resolution in the streamwise direction is $2<\Delta s^{+}<100$ and $2<\Delta l^{+}<30$ in the direction parallel to the surface of the body and normal to the streamwise direction, respectively. The fine grids have $2<\Delta s^{+}<70$ and $2<\Delta l^{+}<20$. Here, $\Delta n^{+}=n\left\langle u_{\tau}\right\rangle_{t} / \nu$, $\Delta s^{+}=\Delta s\left\langle u_{\tau}\right\rangle_{t} / \nu, \Delta l^{+}=\Delta l\left\langle u_{\tau}\right\rangle_{t} / \nu$ and $\left\langle u_{\tau}\right\rangle_{t}$ is the time-averaged friction velocity. The time step in the coarse grid simulations was $5 \cdot 10^{-5} \mathrm{~s}$ and in the fine grid simulations $4 \cdot 10^{-5} \mathrm{~s}$ giving a CFL number below one in all of the simulations. All the cases were initially run for a physical time of 1 second to let the flow develop. This corresponds to a fluid particle travelling the length of the entire numerical wind tunnel 2.5 times. Time averaging started after the initial developing period. The filtered variables were averaged over some convective time of $t \cdot U_{\infty} / L=100$, where $L$ is the length of the model. This corresponds to a fluid particle travelling approximately 11 times through the wind tunnel.

\section{Results}

The denotations that are used in this section for the different parts of the geometry are shown in Fig. 5. The base face of the rear box, denoted RB_BF, is not seen in the figure. The four different cases, Cases 1 - 4 are listed in Table 1. 'Sharp model' refers to the cases with sharp leading edges on the front box and 'round model' refers to the cases with rounded leading edges on the front box. 


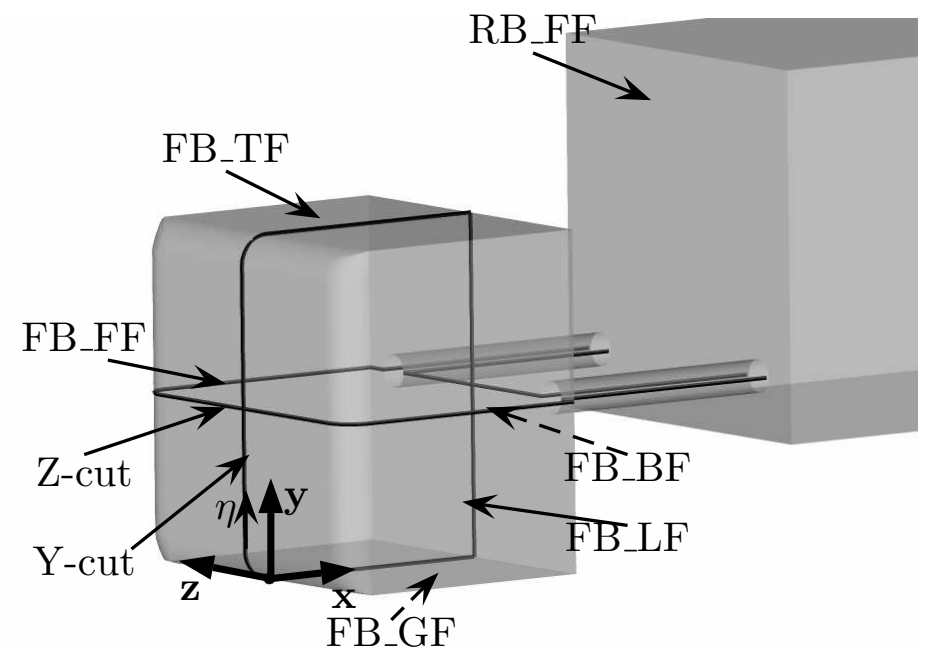

Figure 5: Denotations of parts of the model used in the present work. Coordinate $\eta$ is used to plot the pressure coefficient $\left(C_{P}\right)$ and starts at origo and goes clockwise around the symmetry line on the front box. FB_FF, FB_TF, FB_BF, FB_LF, FB_GF and RB_FF denote the front face of the front box, the top face of the front box, the base face of the front box, the lateral face of the front box, the face closest to the ground and the front face of the rear box, respectively. Y-cut cuts the front box at half of its width $(y=0)$ and is the symmetry plane. Z-cut cuts the front box at $z=0.46 b$.

Table 1: The four different cases studied in the present work.

\begin{tabular}{|l|c|c|}
\hline \hline Name & $\begin{array}{c}\text { Leading edge on } \\
\text { front box }\end{array}$ & Gap width \\
\hline Case 1 & Sharp & $g / b=0.17$ \\
\hline Case 2 & Round & $g / b=0.17$ \\
\hline Case 3 & Sharp & $g / b=0.67$ \\
\hline Case 4 & Round & $g / b=0.67$ \\
\hline \hline
\end{tabular}




\subsection{Comparisons of pressure coefficient on the front box between grids}

Comparison of pressure coefficient $\left(C_{P}\right)$ between the coarse and fine grids are presented in Fig. 6. $C_{P}$ is defined according to Eq. 7 in which $p$ is the absolute pressure, $p_{\infty}$ is the reference pressure in a cell in the upper, right corner at the inlet and the denominator is the dynamical pressure at the inlet. In the figure $C_{P}$ is plotted on the y-symmetry line (see Fig. $5)$. Only very small differences can be observed between the coarse and fine grids for all the cases on FB_FF, FB_TF and FB_BF. There is a noticeable difference on FB_GF however, especially for Case 1 and Case 3 with sharp leading edges on the front box.

$$
C_{P}=\frac{p-p_{\infty}}{0.5 \cdot \rho U_{\infty}^{2}}
$$



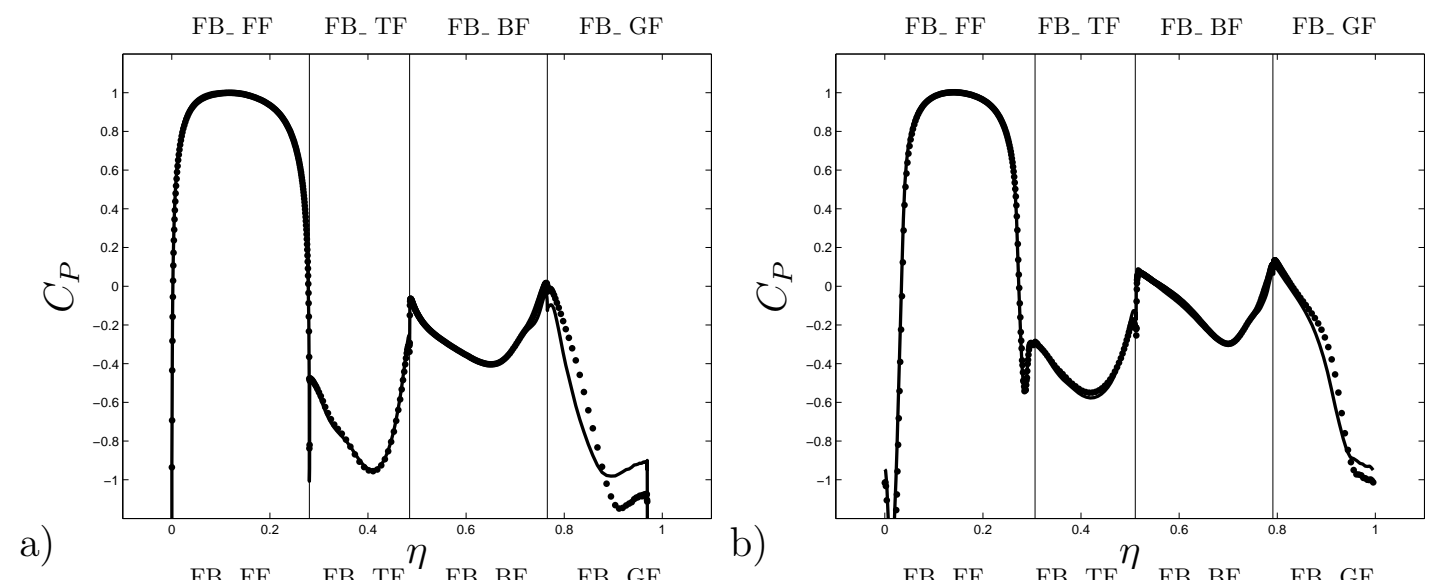

a)

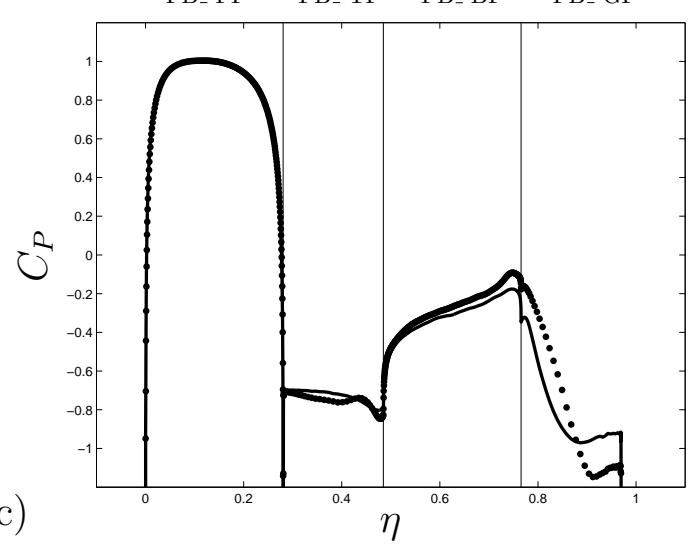

b)

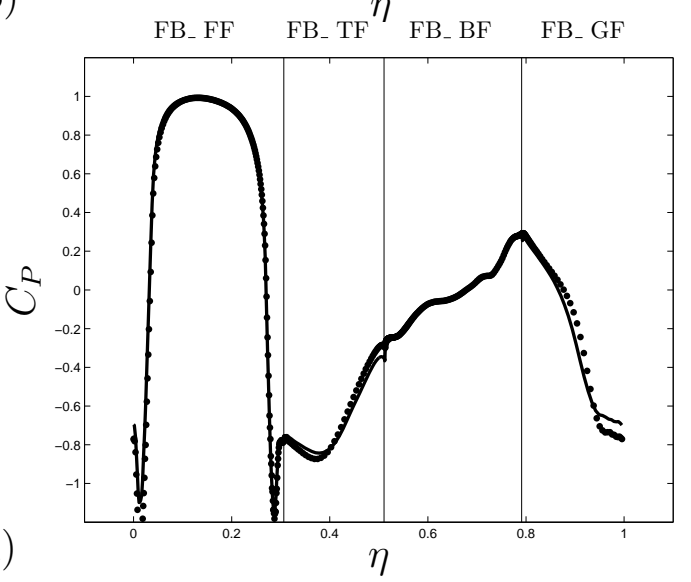

Figure 6: The pressure coefficient $\left(C_{P}\right)$ plotted along the length of the Y-symmetry line on the front box of the model. The solid line shows results from the coarse grid and the dotted line from the fine grid, respectively. The vertical thin drawn lines mark the end of one face and beginning of the next. a) Case 1 (small gap width, sharp edges). b) Case 2 (small gap width, round edges). c) Case 3 (large gap width, sharp edges). d) Case 4 (large gap width, round edges).

\subsection{Time-averaged forces}

The drag coefficients defined according to Eq. 8 are given in Table 2. The only case for which the present LES simulations predict the value of $C_{D}$ in agreement with the experimental data is Case 1, for both the coarse and fine grids. The fine grid for Case 2 yields a value fairly close to that of the experiments (0.793 in simulation, 0.77 in experiment). For Case 3 , the value is overpredicted by $6 \%$ for both of the grids and the value is underpredicted by $12 \%$ and $10 \%$ for the fine and coarse grid, respectively, for Case 4.

$$
C_{D}=\frac{F_{X}}{0.5 \cdot \rho U_{\infty}^{2} b^{2}}
$$


Table 2: Force coefficients results from the simulations together with the available experimental data from Allan [1981]. $\left|\Delta C_{D}\right|$ is the difference between the computed and the experimental value.

\begin{tabular}{|l|c|c|c|}
\hline \hline Aerodynamic coefficients & $C_{D}$ & $r m s\left(C_{D}\right)$ & $\left|\Delta C_{D}\right|$ \\
\hline Case 1 exp & 1.02 & - & - \\
\hline Case 1 fine & 1.023 & 0.041 & $<1 \%$ \\
\hline Case 1 coarse & 1.022 & 0.039 & $<1 \%$ \\
\hline \hline Case 2 exp & 0.77 & - & \\
\hline Case 2 fine & 0.793 & 0.056 & $3 \%$ \\
\hline Case 2 coarse & 0.823 & 0.044 & $7 \%$ \\
\hline \hline Case 3 exp & 1.09 & - & - \\
\hline Case 3 fine & 1.160 & 0.084 & $6 \%$ \\
\hline Case 3 coarse & 1.157 & 0.077 & $6 \%$ \\
\hline \hline Case 4 exp & 1.28 & - & - \\
\hline Case 4 fine & 1.124 & 0.041 & $12 \%$ \\
\hline Case 4 coarse & 1.146 & 0.048 & $10 \%$ \\
\hline \hline
\end{tabular}

The contributions to $C_{D}$ of the entire model from the four contributing faces are shown in Fig. 7. In Allan [1981] the contributions to $C_{D}$ from FB_FF and RB_BF are said to be almost constant over different gap widths for both the sharp and round models. This is also true in the present simulations, which can be seen in Figs. 7a,d. The changes in $C_{D}$ for the entire model when the gap width is varied are thus only due to changes in the contributions from the two gap faces, FB_BF and RB_FF. Much can be understood about the difference between the models (sharp and round leading edges on the front box) by looking at the graphs in Fig. 7. The rounding dramatically reduces $C_{D}$ on FB_FF by some $55 \%$ (see Fig. 7a.) for both gap widths (as expected when rounding the front edge of a prismatic body, see Cooper [1985]). $C_{D}$ is however also reduced by some $50 \%$ on the base face of the front box (FB_BF) with round front edges on the front box for both gap widths (see in Fig. 7b). This was found in both the present numerical study and the experimental study by Allan [1981]. This is a result of the proximity of the rear box to the front box. The large gain in decreased drag for the entire model when the edges of the front box are rounded and the gap width kept small is lost due to an increase in the contribution to the drag from the rear box for large gap widths. The flow mechanism behind this will be explained in Section 3.3 and 3.4. Figure 7c shows the contribution to $C_{D}$ from RB_FF for the sharp and round models, respectively. On the sharp model, there is a negative contribution to $C_{D}$ from RB_FF in both the present simulations and experiments for both gap widths. For the round model with a small gap width, there is a slightly positive contribution to $C_{D}$ from RB_FF. The contribution to $C_{D}$ from RB_FF increases a great deal as the gap width is increased to $g / b=0.67$ for the round model. Thus, starting with Case $1(g / b=0.17$ and sharp leading edges), the decrease in $C_{D}$ that is gained by rounding the leading edges on the front box is lost almost entirely due to the contribution to $C_{D}$ from the front face of the rear box (RB_FF) when the gap width is increased. 
+ Sharp model (Coarse)

X Sharp model (Fine)

* Round model (Coarse)

- Round model (Fine)

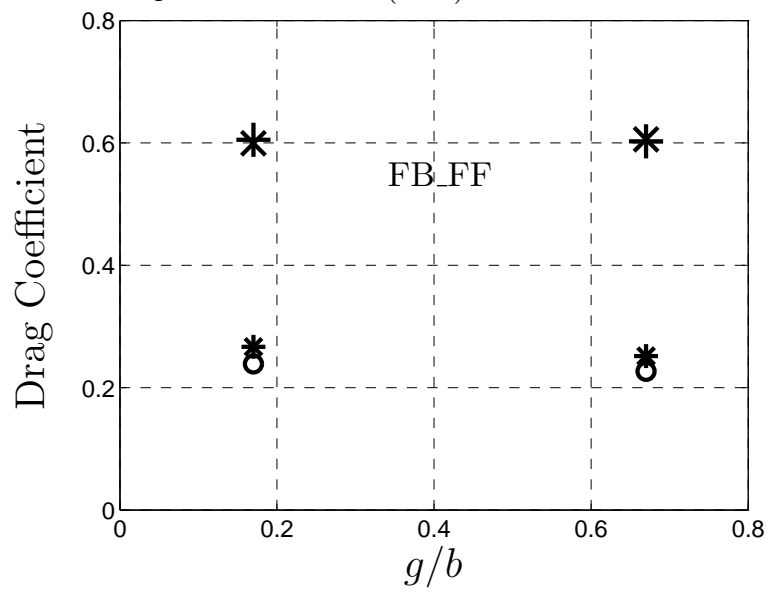

a)

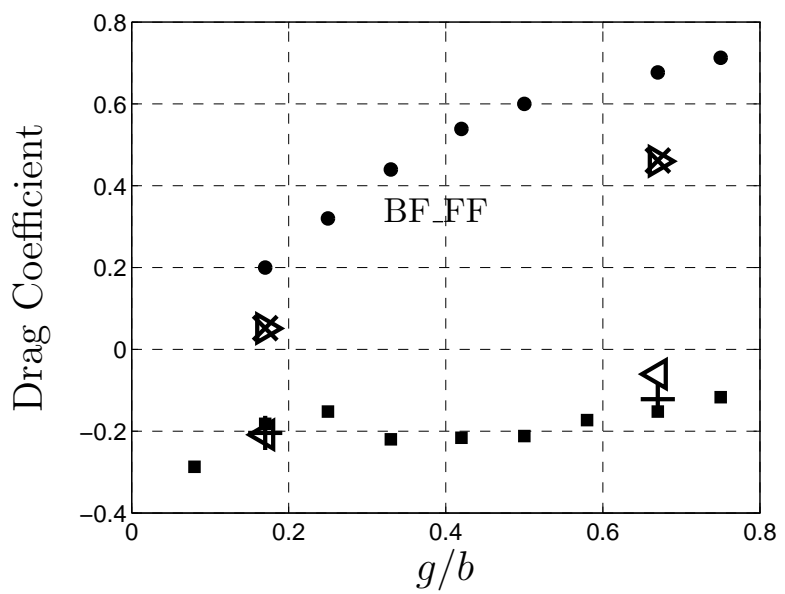

+ Sharp model (Coarse)

$\times$ Round model (Coarse)

$\checkmark$ Sharp model (Fine)

$\triangle$ Round model (Fine)

- Sharp model (Exp)

- Round model (Exp)

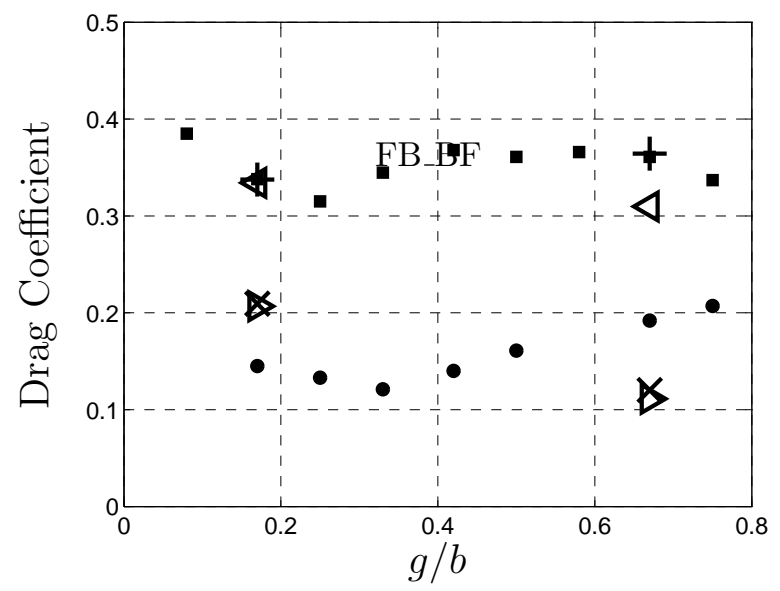

b)

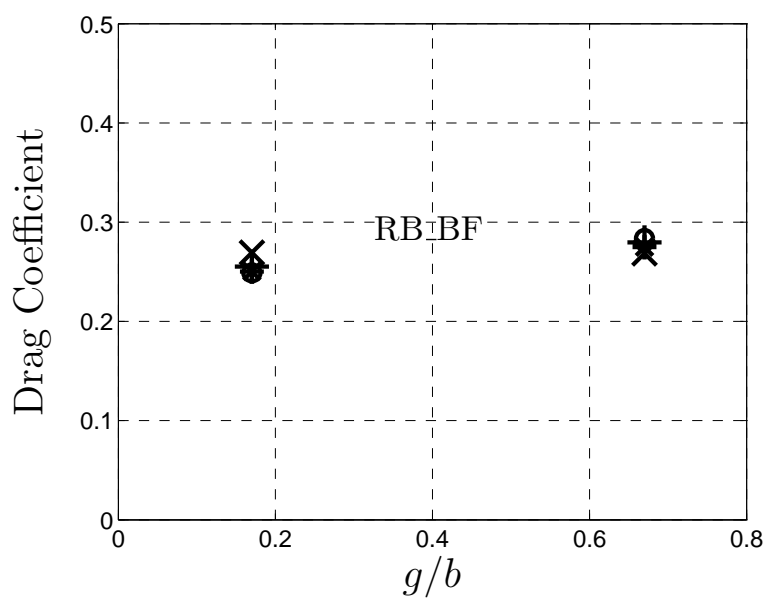

Figure 7: Contribution to the drag coefficent of the entire model from the four different contributing faces. a) FB_FF. b) FB_BF c) RB_FF. d) RB_BF. Note that in a) and d) only results from the coarse and fine grid simulations are presented since there were no experimental results available for these faces. 


\subsection{Time-averaged flow structures}

The time-averaged flow fields around the models are presented in this section and all the results shown come from the fine grid simulations. The four cases (Cases 1-4) will be subdivided into the groups "small gap width" and "large gap width" to emphasize the difference in the flow structures that emerges by rounding the leading edges of the front box.

\subsubsection{Small gap width}

This section presents the mean flow around the two cases (Case 1 and Case 2) with a small gap width $(g / b=0.17)$. Case 1 has a sharp leading edge on the front box and Case 2 has a rounded. Vortex cores are computed by the algorithm in the EnSight visualization package. The method for vortex core identification is based on critical-point concepts for fluid flow (e.g Perry and Chong [1987]) and finds eigenvalues and eigenvectors to the rate-of-deformation tensor of the filtered velocity, $\partial \bar{u}_{i} / \partial x_{j}$. A full description of the algorithm is given by Sujudi and Haimes [1995]. The algorithm can produce non-existing cores and should only be used as a tool to help localize vortices (Sujudi and Haimes [1995]). Streamlines around cores are used in the present work to assure that the cores really exist. The vortical structures that are of interest to us in this work are large vortical structures in the order of magnitude of $b$ caused by separation of the flow at leading and base edges. Streamlines of the time-averaged velocity projected onto the surface are also used to reveal features in the flow. The streamline pattern on the surface will reveal points where the streamline slope is indeterminate (i.e. all the spatial derivatives of the velocity are zero). These points are called critical points (Perry and Chong [1987]) and by interpreting the pattern of these points the salient features in the flow can be understood. Critical points and bifurcation lines are shown in Fig. 8. Three kinds of critical points can be formed: nodes, foci and saddles (see. Fig. 8). The first two can be either stable or unstable. Other patterns that the streamlines form are negative (NBL) and positive (PBL) bifurcation lines. NBLs are associated with flow separation and PBLs are associated with flow attachment. For an example of how streamline patterns have been used previously in vehicle aerodynamics we refer to Krajnović and Davidson [2005b]. 


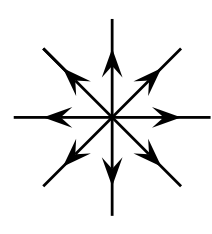

$\mathrm{UN}$

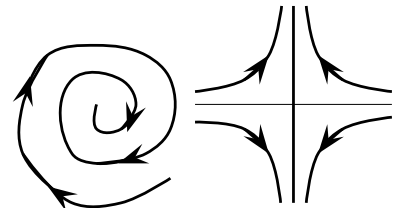

$\mathrm{SF}$ SP

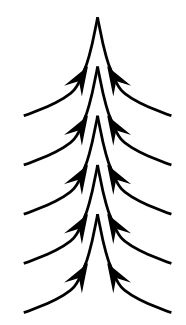

NBL

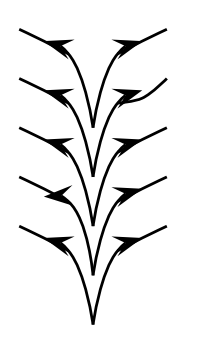

PBL

Figure 8: Critical points and bifurcation lines. SP is a saddle point, SF is a stable focus, UN is an unstable node, NBL is a negative bifurcation line and PBL is a positive bifurcation line. 


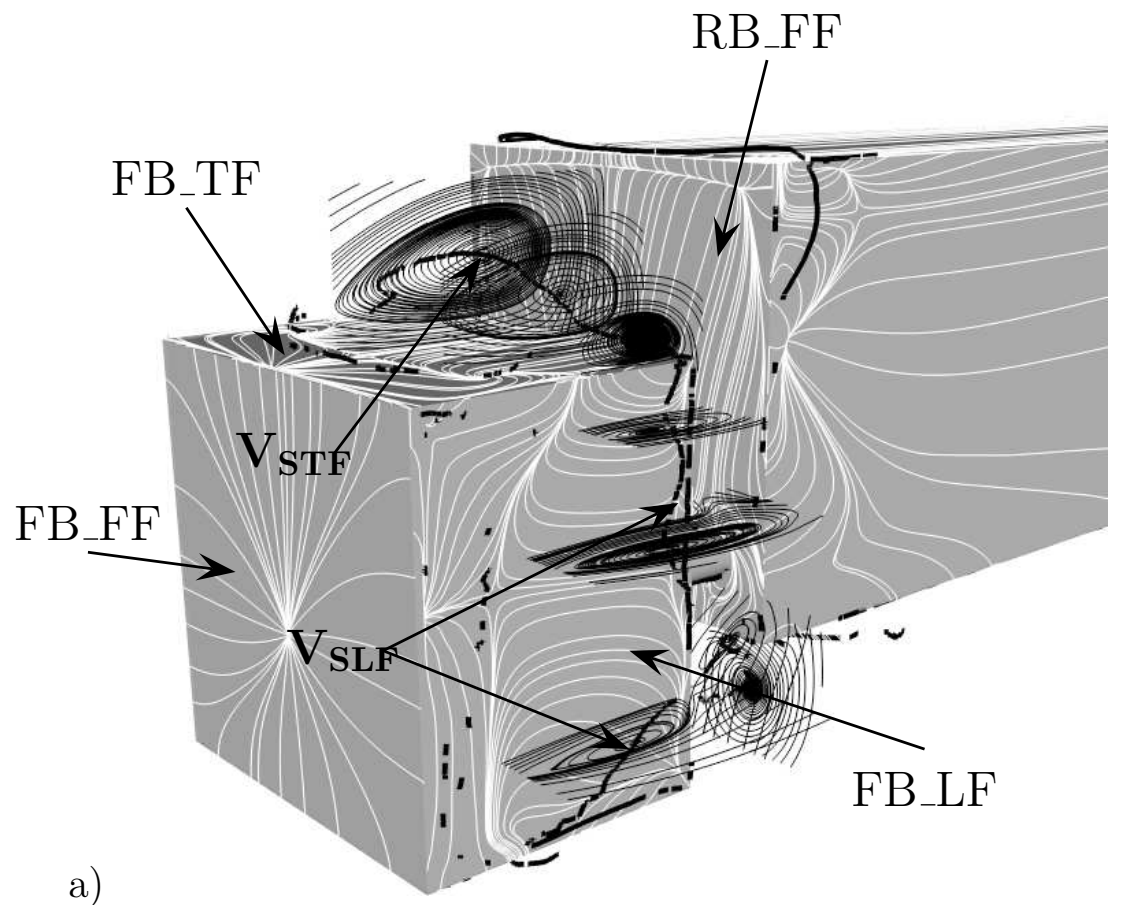

a)

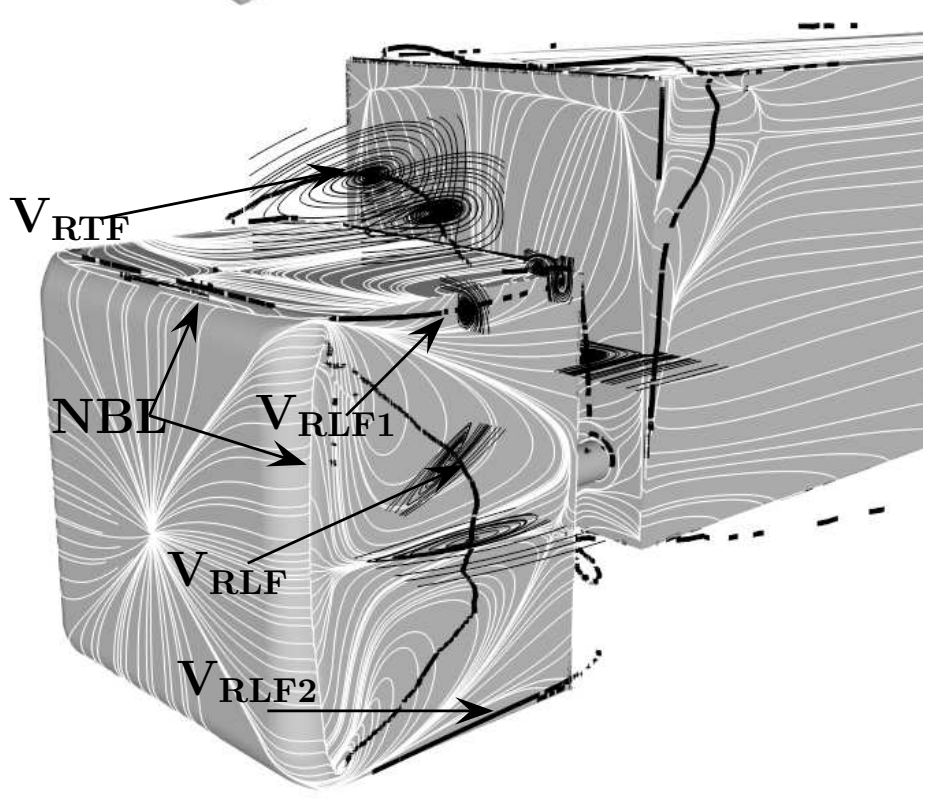

b)

Figure 9: Streamlines of the time-averaged velocity projected onto the surface of the body (white lines) together with vortex cores (black drawn lines) and streamlines projected onto planes around vortex cores (black lines). a) Case 1 (small gap width, sharp edges). b) Case 2 (small gap width, round edges) 
Time-averaged flow structures around the cab are presented for Case 1 in Fig. 9a and Case 2 in Fig. 9b. In the figures, the white lines are streamlines of the time-averaged velocity projected onto the surface of the body. The black streamlines of the time-averaged velocity are projected onto planes around the vortex cores (solid black lines). For both cases, the free-streaming air hits the front face of the front box (FB_FF) and separates at the leading edges. Over the top face of the front box $\left(\mathrm{FB}_{-} \mathrm{TF}\right)$ the flow separations form vortices $V_{S T F}$ (see Figs. 9a and 16a) and $V_{R T F}$ (see Figs. 9b and 16b) for Case 1 (sharp model) and Case 2 (round model), respectively. Outside of the lateral face of the front box (FB_LF) for Case 1 the vortex $V_{S L F}$ is formed which seems to be connected to the vortex over the top face, $V_{S T F}$. The vortex formed outside FB_LF is called $V_{R L F}$ (see Fig. 9b) for Case 2 , and it is not connected with $V_{R T F}$ which is formed over FB_TF (see Fig. 9b). Another difference between Case 1 and Case 2 is the trailing vortices, $V_{R L F 1}$ and $V_{R L F 2}$ (see Fig. 9b), that extend in a streamwise direction along the upper and lower edges on FB_LF for Case 2. Similar type of trailing vortices as $V_{R L F 1}$ and $V_{R L F 2}$ were not found for Case 1 where the flow around the front box is dominated by the vortices $V_{S T F}$ and $V_{S L F}$ (see Fig. 9a). Figure 10 shows streamlines of the time-averaged velocity projected onto plane $z=0.46 b$ for Cases 1 - 4. For Case 1 (sharp model) the flow does not re-attach on the lateral face of the front box (FB_LF) after first separating on the leading edge on the front box (see Fig. 10a). The large vortex $V_{S L F}$ which is formed due to the flow separation on the leading edge of the front box, extends over the whole gap and the flow re-attaches on the lateral face of the rear box (see Fig. 10a). For Case 2 (round model) the flow does re-attach on FB_LF. This is shown in Fig. 10b and by the surface-projected streamlines on the body in Fig. 9b. 


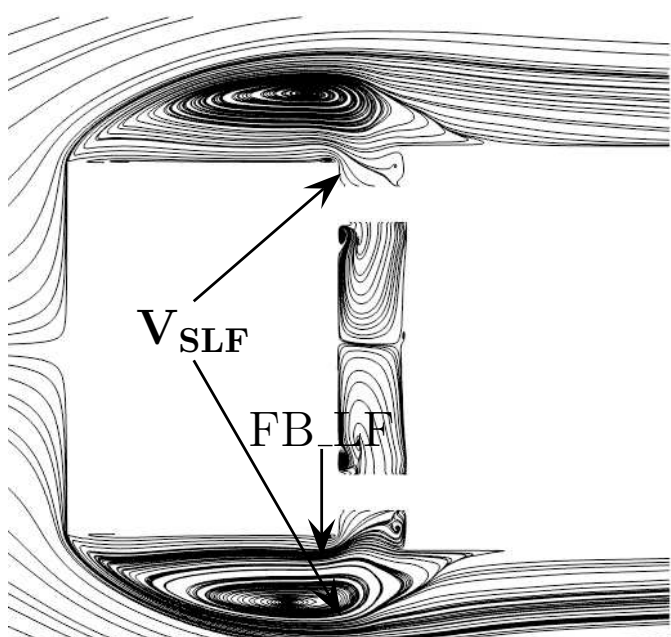

a)

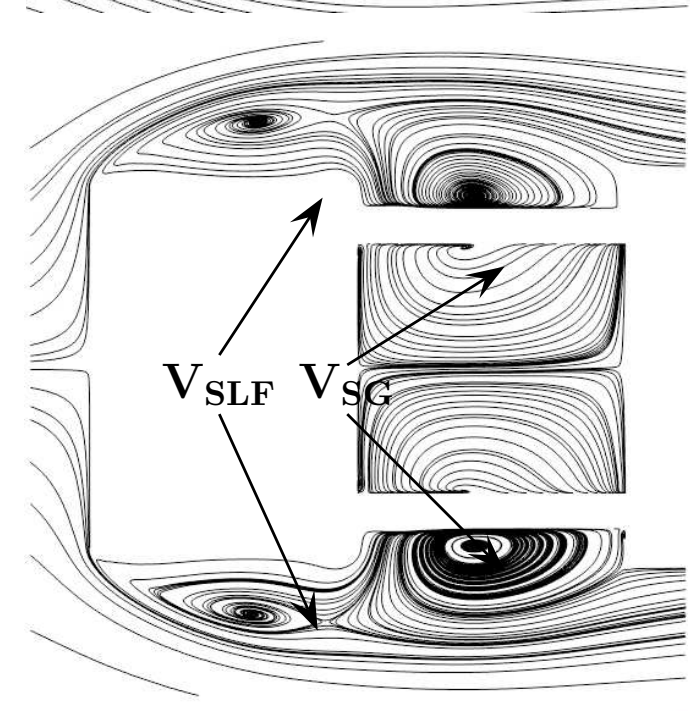

b)

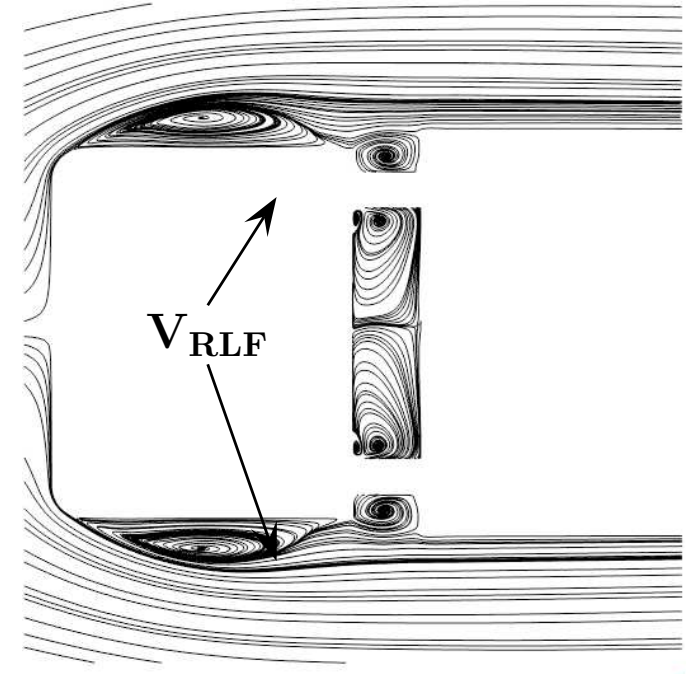

d)

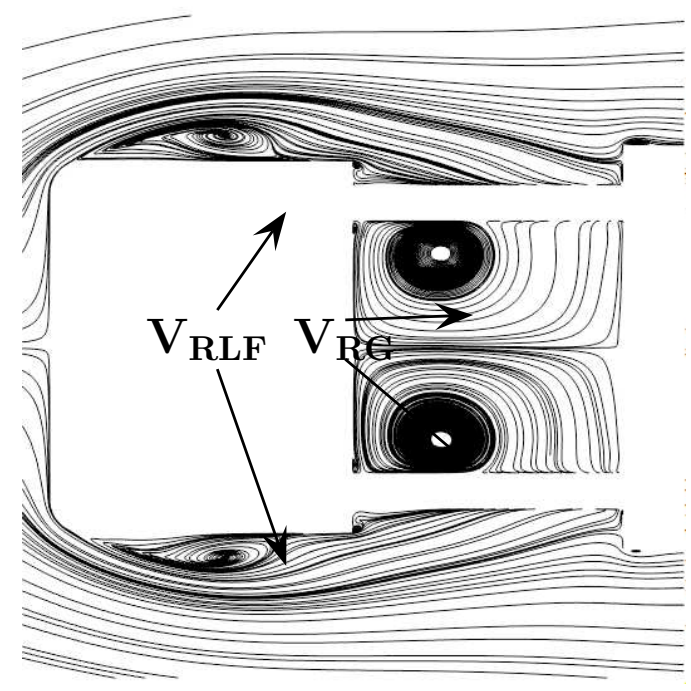

Figure 10: Streamlines of the time-averaged velocity projected onto plane $z=0.46 b$. a) Case 1 (small gap width, sharp edges). b) Case 2 (small gap width, round edges). c) Case 3 (large gap width, sharp edges). d) Case 4 (large gap width, round edges). 

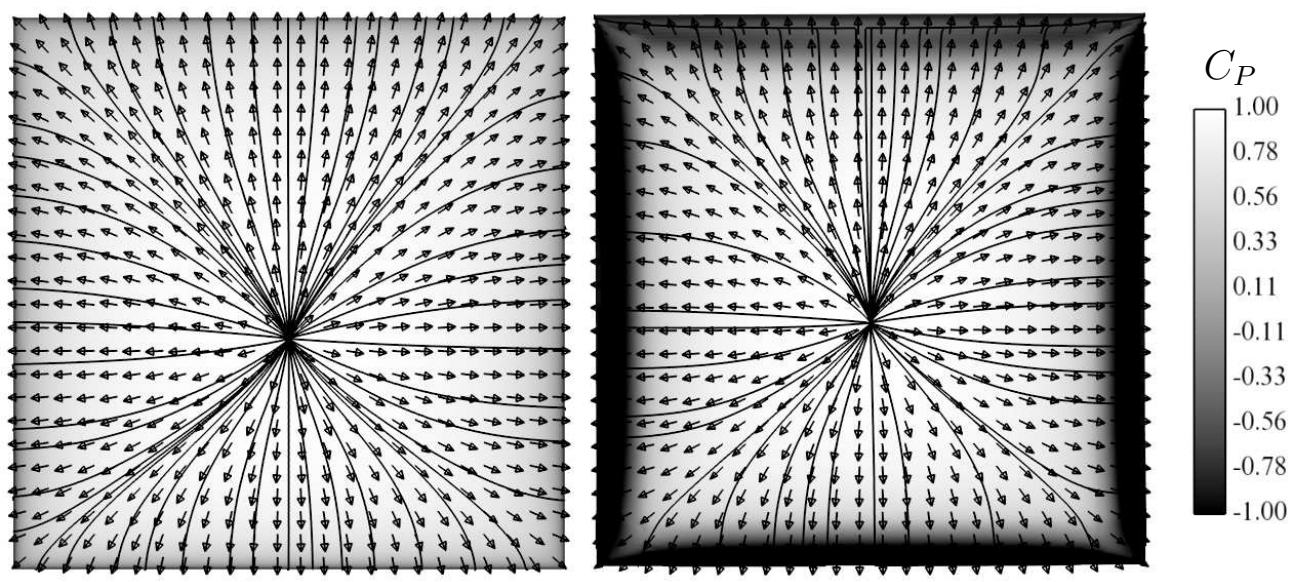

Figure 11: Pressure coefficient on FB_FF together with streamlines of the time-averaged velocity projected onto the surface. Left: Case 1 (small gap width, sharp edges). Right: Case 2 (small gap width, round edges)

Figure 11 shows streamlines of the time-averaged velocity projected onto the front face of the front box (FB_FF) together with values of $C_{P}$ on the surface for Case 1 and Case 2 . The sharp model (Case 1) is characterized by positive pressure everywhere on FB_FF and a value of $C_{P}$ close to one (see Fig. 11), which is typical for stagnation points. On the round model the air accelerates over the curvature at the edges before separating. This separation of the flow is marked by the negative bifurcation lines (NBL) in Fig. 9b. The acceleration of the air over the curvature gives rise to the area of low pressure distinguished by the black part around the edges in Fig. 11. This explains the large decrease in $C_{D}$ on FB_FF (see Fig. 7b) when the leading edges are rounded.
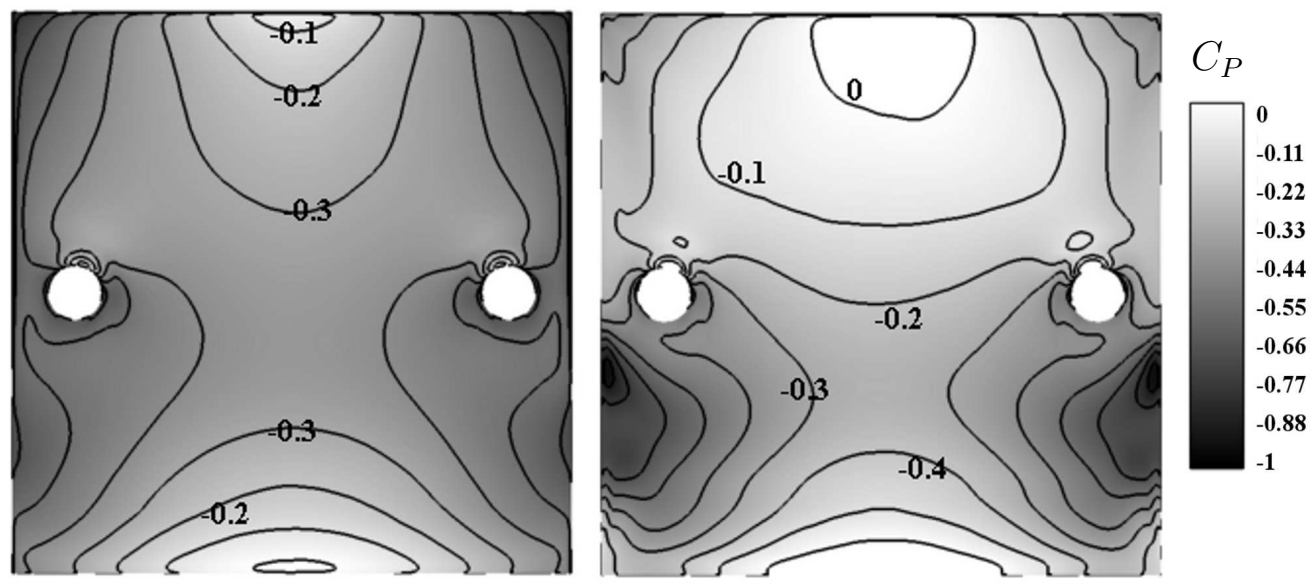

Figure 12: Pressure coefficient on FB_BF. Please note that the span of values is from - 1 to 0 and not from -1 to 1 as in Fig. 11. Left: Case 1 (small gap width, sharp edges). Right: Case 2 (small gap width, round edges)

The pressure coefficients on the base face of the front box (FB_BF) are shown in Fig. 12 for Case 1 and Case 2. As seen in Fig. 7b) the contribution to $C_{D}$ from FB_BF for Case 
2 is some $30 \%$ less than for Case 1 ( 0.34 for Case 1 and 0.21 for Case 2). The primary reason for this is that the pressure along the lateral edges on the face for Case 1 is lower than for Case 2 (see Fig. 12). The reason why the pressure along the lateral edges is lower for Case 1 than for Case 2 is the differences in the strength and extension of the vortices outside of the lateral face of the front box for the two cases. For Case 1, the vertical part of $V_{S L F}$ outside FB_LF (see Figs. 9a and 10a) extends all over the gap. $V_{S L F}$ creates very low pressure all over FB_LF and, since the vortex extends over the gap, the low pressure pulls into the gap and conduces to low pressure not only on FB_BF but on the front face of the rear box (RB_FF) as well. For Case 2, the vertical vortex $V_{R L F}$ outside FB_LF (see Figs. $9 \mathrm{~b}$ and $10 \mathrm{~b}$ ) does not extend over the gap, but re-attaches on FB_LF. This conduces to a much higher pressure on the right part of FB_LF and thus higher pressure in the gap and on the base face of the front box (FB_BF). Figure 13 shows $C_{P}$ on FB_LF for Case 1 (left) and Case 2 (right). Note the large difference in the pressure on the face due to the different nature of the vortices $V_{S L F}$ (Case 1) and $V_{R L F}$ (Case 2), as discussed above.
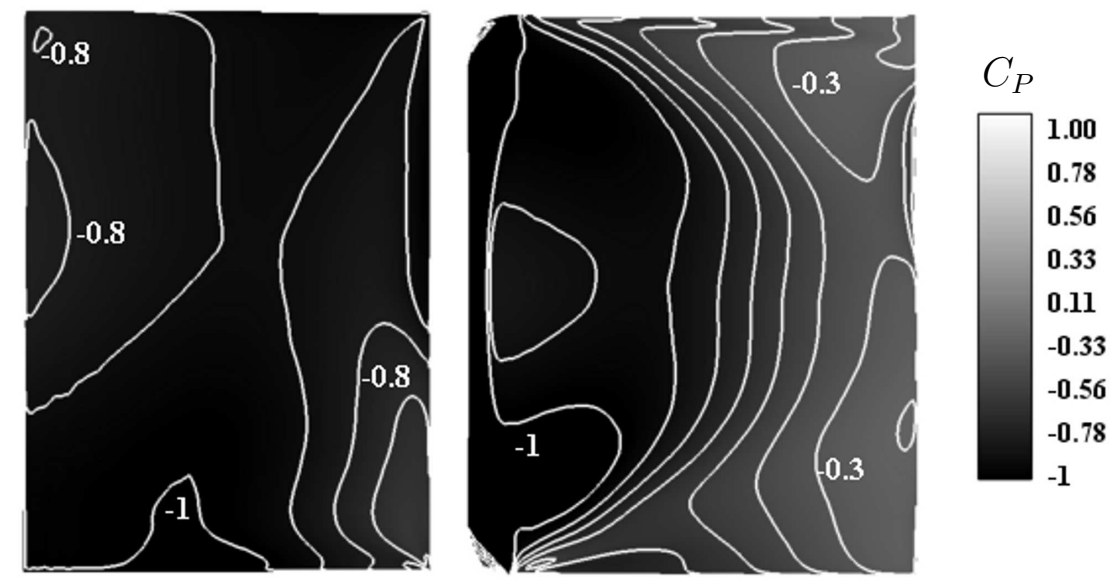

Figure 13: Pressure coefficient on FB_LF. Left: Case 1 (small gap width, sharp edges). Right: Case 2 (small gap width, round edges)

The net force on the front face of the rear box (RB_FF) is negative and in a direction opposite to the direction of the flow for Case 1 (see Fig. 7c). It is slightly positive (see Fig. $7 \mathrm{c}$ ) for Case 2 (round model). Figure 14 shows $C_{P}$ for Case 1 and Case 2 on RB_FF. There is lower pressure for Case 1 for the lower half of the face compared with Case 2. This is again explained by the extension over the gap of the vertical part of vortex $V_{S L F}$ (see Figs. $9 \mathrm{a}$ and 10a) compared with the smaller extension of the vortex $V_{R L F}$ for Case 2 (see Figs. 9b and $10 \mathrm{~b}) . V_{R L F}$ does not extend over the gap. For Case 1, $V_{S L F}$ shields the vertical edge of RB_FF from the airstream, and thus a low pressure is kept all the way to the outermost part of the face in a spanwise sense. $V_{R L F}$ is not able to shield all of RB_FF for Case 2, a part of the airstream impinges on the outermost part of the face and high pressure is induced there (see Fig. 14). The high pressure contributes to an increase in the drag coefficient for Case 2 . 

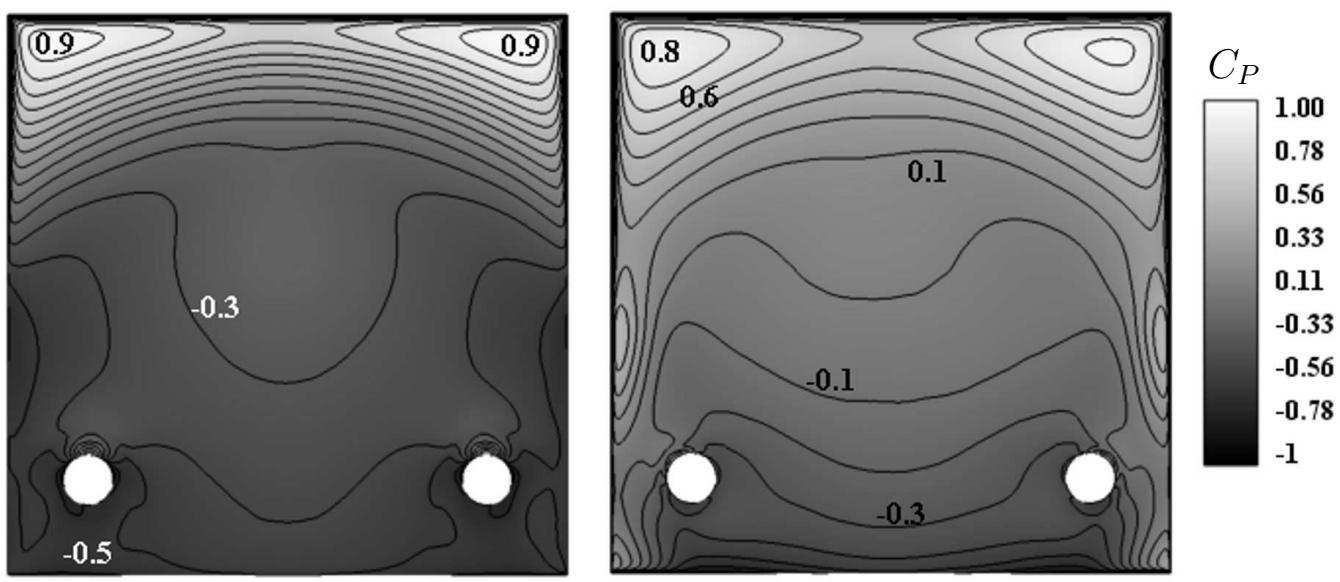

Figure 14: Pressure coefficient on RB_FF. Left: Case 1 (small gap width, sharp edges). Right: Case 2 (small gap width, round edges).

\subsection{Large gap width}

This section describes the mean flow fields for the two cases with a large gap width (Case 3 and Case 4). Some similarities and differences in the flow between small and large gap widths are noted and analysed for the sharp and round models, respectively. 


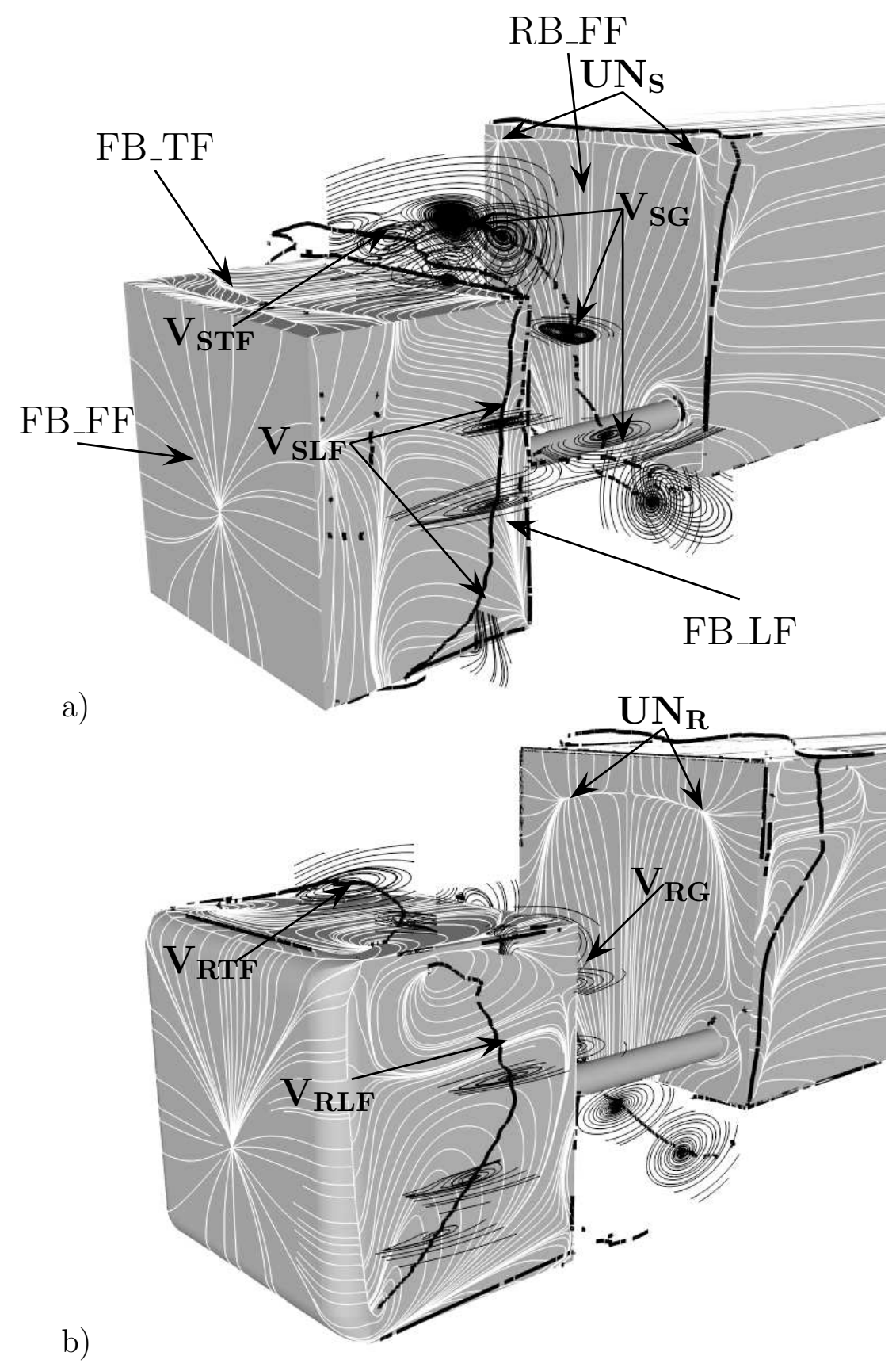

Figure 15: Streamlines of the time-averaged velocity projected onto the surface of the body (white lines) together with vortex cores (black drawn lines) and streamlines projected onto planes around vortex cores (black lines). a) Case 3 (large gap width, sharp edges). b) Case 4 (large gap width, round edges). 
Figure $15 \mathrm{a}$ shows the system of vortical structures of the mean flow around Case 3 $(g / b=0.67$, sharp edges). Over the top face of the front box (FB_TF) there's a type of vortex similar to $V_{S T F}$ for Case 1 (see Fig. 9a). The vortex over FB_TF for Case 3 is called $V_{S T F}$ as well in Fig. 15a. The vortex outside of the lateral face of the front box (FB_LF) for Case 3 is called $V_{S L F}$ in Fig. 15a. As seen by comparing streamlines in plane $z=0.46 \mathrm{~b}$ in Figs. 10a (Case 1) and 10c (Case 3), vortex $V_{S G}$ is formed in the gap for Case $3(\mathrm{~g} / \mathrm{b}=0.67$, sharp edges). Vortex $V_{S G}$ is also pointed out in Fig. 15a, and it is a large horse-shoe like vortex. It extends horizontally in the gap and then down vertically in two tails outside the two connecting cylinders between the front and rear boxes. The mean flow in the gap is completely dominated by this vortex for Case 3. $V_{S G}$ (see Fig. 15a) is responsible for the low pressure on FB_BF (see Fig. 7a), contributing to an increase in $C_{D}$ for the whole model for Case 3. However, $V_{S G}$ is also responsible for the low pressure on the front face of the rear box (RB_FF, see Fig. 7c) which contributes to a decrease in $C_{D}$ for the entire model.

Figure 15b shows the vortex system around the model for Case $4(g / b=0.67$, round edges). The vortex outside of FB_LF for Case $4\left(V_{R L F}\right)$ is very similar to vortex $V_{R L F}$ (see Fig. $9 b)$ outside FB_LF for Case $2(g / b=0.17$, round edges). The vortex above FB_TF for Case 4 is called $V_{R T F}$ in Fig. 15b. For Case 4 there is also a horse-shoe like vortex, $V_{R G}$, that forms in the gap (see Fig. 15b), as for Case 3. Topologically, there is no large difference in the flow in the gap for the two cases with large gap width. The gap is for both cases dominated by the horse-shoe like vortices $V_{S G}$ and $V_{R G}$, respectively (see streamlines in plane $z=0.46 b$ in Figs. 10c and 10d). The vertical tails of $V_{R G}$ are however confined to the space between the connecting cylinders between the front and rear boxes (see Fig. 10d) while the tails of $V_{S G}$ are located outside the cylinders in a spanwise sense (see Figs. $10 \mathrm{c}$ and 15a). $C_{P}$ for Case 3 and Case 4 on RB_FF is presented in Fig. 17. Note the much higher pressure for Case 4 than for Case 3 on RB_FF, indicating that $V_{R G}$ is weaker than $V_{S G}$. 
a)

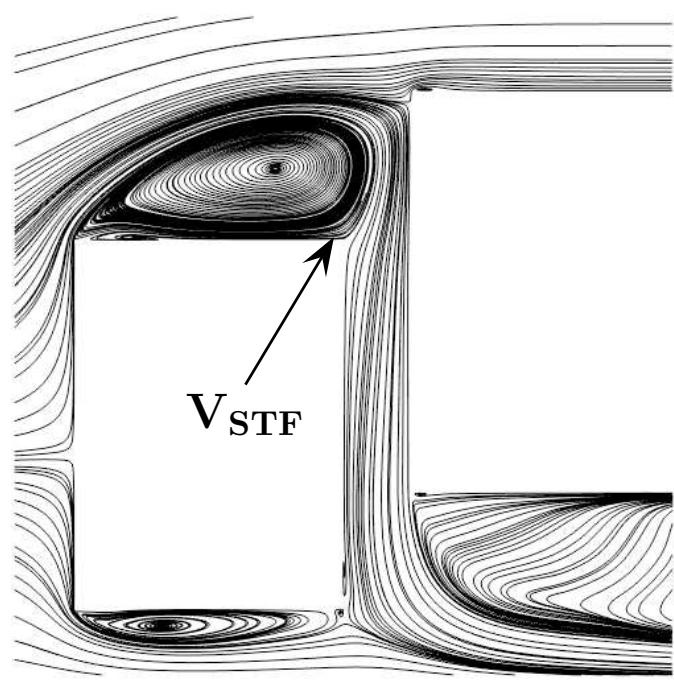

c)

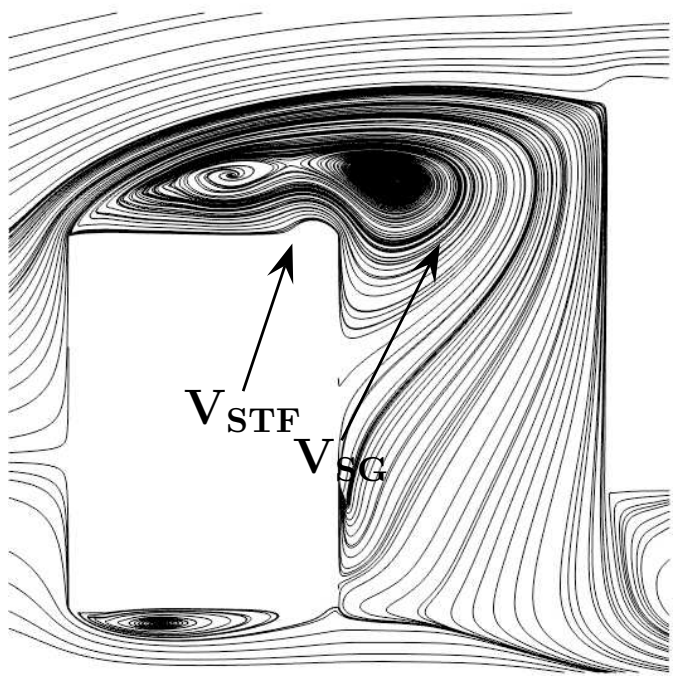

b)

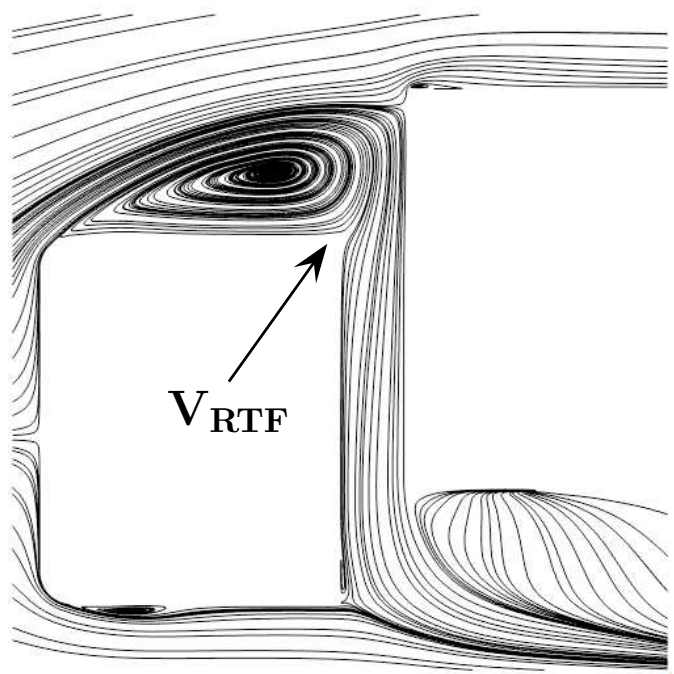

d)

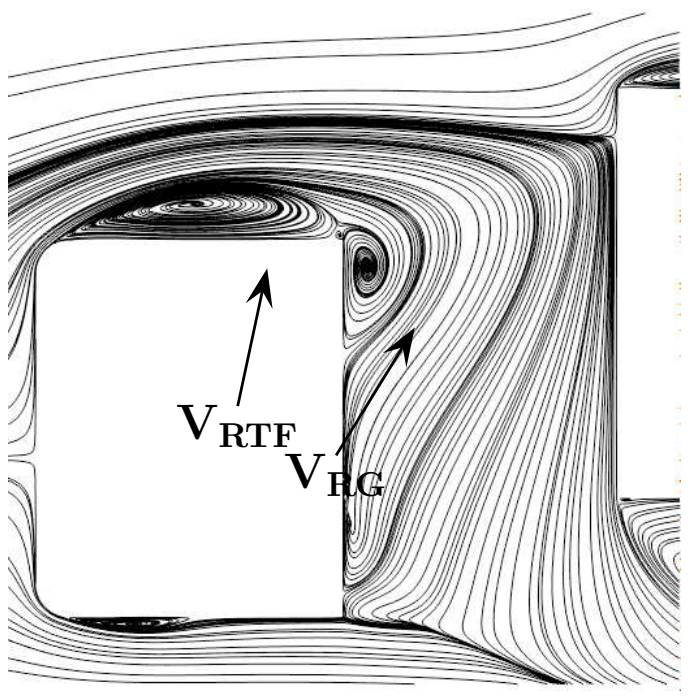

Figure 16: Streamlines of the time-averaged velocity projected on plane y =0. a) Case 1 (small gap width, sharp edges). b) Case 2 (small gap width, round edges). c) Case 3 (large gap width, sharp edges). d) Case 4 (large gap width, round edges). 


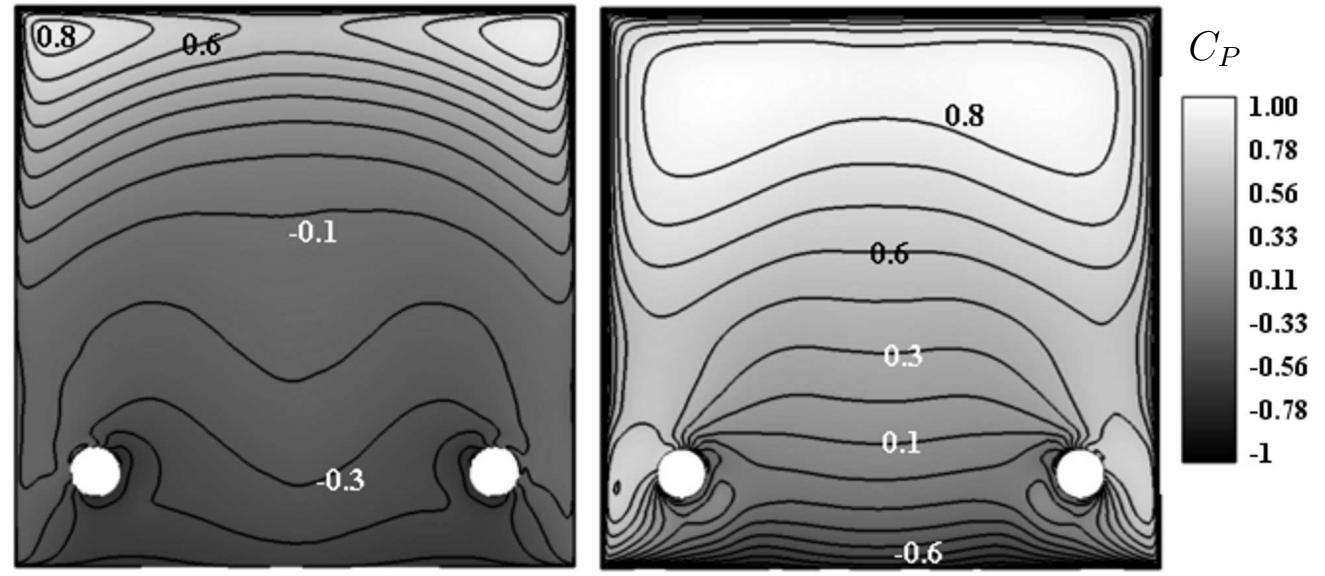

Figure 17: Pressure coefficient on RB_FF. Left: Case 3 (large gap width, sharp model). Right: Case 4 (large gap width, round model). 
The difference between the contributions to $C_{D}$ (see Fig. 7c) from the front face of the rear box (RB_FF) between Case 3 and Case 4 is very large. Figure $7 \mathrm{c}$ shows that the contribution is slightly negative for Case $3(-0.06)$. The contribution to $C_{D}$ from RB_FF for Case 4 is 0.467 . The reason why the contributions from RB_FF are so different between Case 3 and Case 4 is the difference in the vortices formed in the gap in the two cases, $V_{S G}$ for Case 3 (see Fig. 15a) and $V_{R G}$ for Case 4 (see Fig. 15b). Since the only geometrical difference between the Case 3 and Case 4 is the rounding of the leading edge on the front box, the large disparities between the cases in the gap flow are interesting. The difference is that, for Case 4, since $V_{R G}$ is confined between the connecting cylinders, it is not able to shield RB_FF from the airstream flowing along the lateral sides. For Case 3, the gap vortex, $V_{S G}$, is located outside of the connecting cylinders and is much larger and stronger. This can be seen by comparing the streamlines around the vortices in plane $z=0.46 b$ (see Figs. 10c and 10d). Furthermore, the foci of $V_{S G}$ are also located further away from the base part of the front box compared with the tails of $V_{R G}$.

The above described difference between $V_{S G}$ and $V_{R G}$ has the consequence that, for Case 3, the gap vortex, $V_{S G}$, is able to shield the exposed face, RB_FF, from the airstream impinging, and thus a low pressure is kept in the gap and the contribution to $C_{D}$ from $\mathrm{RB} \_\mathrm{FF}$ is negative for Case 3. For Case 4, however, the gap vortex $V_{R G}$ is not able to shield the front face of the rear box (RB_FF) and the airstream impinges on a larger area of RB_FF and thus momentum is directly transferred from the free-streaming air to this exposed surface. The airstreams impinging on RB_FF for Case 3 and Case 4 can also be seen by looking at the two unstable nodes located in the upper corners of RB_FF. The unstable nodes for Case 3 are denoted $U N_{S}$ (see Fig. 15a) and for Case $4 U N_{R}$ (see Fig. 15b). $U N_{R}$ (Case 4) are located closer to the midpoint of the face compared with the location of $U N_{S}$ (Case 3 ). Thus the vertical parts (the tails) of $V_{S G}$ (Case 3) are able to shield the exposed face, RB_FF, and direct the airstream around the face so that the air attaches on the lateral face of the rear box instead. There is another fundemental difference between gap vortices $V_{S G}$ and $V_{R G}$. The former vortex is a result of the air separating on the leading edge on the front box. This can be understood by looking at the streamlines projected in plane $z=46 b$ in Fig. 10c) and in plane $y=0$ in Fig. 16c. $V_{R G}$ is a result of the air separating on the base edge of the front box (see Figs. 10d) and 16d). The air separates on the leading edge, re-attaches on the right part of FB_LF and then separates again on the base lateral edge of the front box, and $V_{R G}$ is formed. The free-streaming air that flows outside of the FB_LF vortex $V_{R L F}$ (see Fig. 15b) then confines $V_{R G}$ to the area between the connecting cylinders in the gap.

\section{Conclusion}

The LES was successfully employed to solve the flow around a simplified tractor-trailer model. The model consisted of a tractor and a trailer separated by a gap and has previously been investigated experimentally in a wind tunnel (Allan [1981]). Four different cases of the model were studied. Two cases (Case 1 and Case 2) had a small gap width between the 
tractor and the trailer. Case 1 had sharp leading edges on the front box and Case 2 had rounded edges. The roundness of the leading edge was found to decrease the drag coefficient for Case 2 in comparison with Case 1 in the same order of magnitude as in the reference experiments. The other two cases (Case 3 and Case 4) had a large gap width and Case 3 had sharp leading edges on the front box and Case 4 had rounded. The drag coefficient for Case 3 increased slightly compared to the drag coefficient for Case 1 as expected from the experimental results. The drag coefficient for Case 4 was expected to increase dramatically (in experiments, the increase was from 0.77 to 1.28). The increase of the drag coefficient for Case 4 compared with Case 2 was large in the present LES, but not as large as expected (in simulations, the increase was from 0.79 to 1.13). The main reason for this smaller increase in the simulations compared to in the experiments was that the contribution to the drag coefficient of the whole vehicle from the front face of the rear box (RB_FF) was underpredicted in the simulations (0.467 in LES compared with 0.677 in experiments). In general, the results from the two cases with sharp leading edge on the front box was in closer agreement to the experimental values.

The mean flow fields around the four cases were described. For Case 1, the flow around the front box and the gap was dominated by the vortices caused by the separation of the flow on the sharp leading edge on the front box. Almost all of the drag force on the vehicle was taken up by the front box, and the resulting force on the rear box was close to zero. Rounding of the leading edges on the front box reduced the drag force of the front box and thus the whole vehicle for Case 2 for which the gap width was small. The flow field around the front box and the gap for Case 2 was similar to the one around Case 1 but the roundness of the leading edges on the front box caused much smaller vortices due separation than for Case 1. The difference in drag coefficient (1.022 and 0.793 for Case 1 and Case 2, respectively) was found to be mainly due to the decreased pressure along the curvature on the rounded edges on the front box for Case 2. For the large gap width, the gain in decreased drag of the front box when rounding the leading edges was lost due to increased drag of the rear box. It was shown that the reason for this was that the vortex formed outside of the lateral face of the front box in Case 4 was much smaller and could not shield the front face of the rear box of the model from the oncoming flow.

The present work shows that LES can be used to gain knowledge about the flow structures around simplified models of tractor-trailers which includes gap between the tractor and the trailer. This is a rather complex bluff body system that includes a number flow regions which are difficult to predict with CFD. These are e.g. separation of flow from rounded edges, attached boundary layer flow on the trailer and interaction between the tractor and the trailer. The computer expense of the simulations is managable due to the separated character of the flow. Without large regions of attached flow, the LES requirements for near-wall resolution could be relaxed. Even though the Reynolds number in the present study is rather high $\left(0.51 \times 10^{6}\right.$ based on the height of the rear box $)$, it is still one tenth of that of tractor-trailers on roads at operational speeds. With increasing Reynolds numbers, changes in the flow structures can be expected. Especially in the cases with rounded leading 
edges on the front box. It has been seen in the present work that the drag coefficient of the model is very sensitive to the flow structures emanating from the separation from the leading edges. Thus, it can be expected that an increased Reynolds number will have large influence on the drag coefficient as well. The present work shows how the flow structures affect the drag coefficient of the model. The comparison between simulated and experimental drag coefficients showed rather large disparities, especially for Case 4 with round edges on the front box and large gap width. Important knowledge about the flow structures and how they affect the drag coefficient and interact with the tractor and the trailer was obtained.

\section{References}

Allan, J., 1981. Aerodynamic drag and pressure measurements on a simplified tractor-trailer model. Journal of Wind Engineering and Industrial Aerodynamics 9, 125-136.

Arcas, D., Browand, F., Hammache, M., 2004. Flow structure in the gap between two bluff bodies. AIAA Paper 2004-2250.

AVL, 2009. CFD Solver. AVL Fire Manual, v2009.1, edition 04/2009.

Barnard, R. H., 1996. Road Vehicle Aerodynamic Design, An Introduction, 1st Edition. Addison Wesley Longman Limited, ISBN 0-582-24522-2.

Cooper, K. R., 1985. The effect of front-edge rounding and rear edge shaping on the aerodynamic drag of bluff vehicles in ground proximity. SAE Paper No. 850288.

Cooper, K. R., 2002. Commercial vehicle aerodynamic drag reduction: Historical perspective as a guide. In: The Aerodynamics of Heavy Vehicles: Trucks, Busses and Trains. Monterey, USA.

Cooper, K. R., 2003. Truck aerodynamics reborn - lessons from the past. SAE Paper 2003-01-3376.

Ghosal, S., Moin, P., 1995. The basic equations for the large eddy simulation of turbulent flows in complex geometry. Journal of Computational Physics 118, 24-37.

Hammache, M., Browand, F., 2002. On the aerodynamics of tractor-trailers. In: The Aerodynamics of Heavy Vehicles: Trucks, Busses and Trains. Monterey, USA.

Hemida, H., Krajnović, S., Davidson, L., 2005. Large eddy simulations of the flow around a simplified high speed train under the influence of cross-wind. In: 17th AIAA Computational Dynamics Conference. Toronto, Ontario, Canada.

Hucho, W.-H., 1998. Aerodynamics of Road Vehicles, 4th Edition. Society of Automotive Engineers, Inc., ISBN 0-7680-0029-7.

Krajnović, S., 2009. LES of flows around ground vehicles and other bluff bodies. Philosophical Transactions of the Royal Society A 367 (1899), 2917-2930.

Krajnović, S., 2011. Flow around a tall finite cylinder explored by large eddy simulation. Journal of Fluid Mechanics 676, 294-317.

Krajnović, S., Davidson, L., 2003. Numerical study of the flow around the bus-shaped body. ASME: Journal of Fluids Engineering 125, 500-509.

Krajnović, S., Davidson, L., 2005a. Flow around a simplified car, part 1: Large eddy simulation. ASME: Journal of Fluids Engineering 127, 907-918.

Krajnović, S., Davidson, L., 2005b. Flow around a simplified car, part 2: Understanding the flow. ASME: Journal of Fluids Engineering 127, 919-928.

McCallen, R., Flowers, D., T. Dunn, J. O., Browand, F., Hammache, M., Leonard, A., Brady, M., Salari, K., Rutledge, W., Ross, J., Storms, B., Heineck, J. T., Driver, D., Bell, J., Walker, S., Zilliac, G., 2000. Aerodynamic drag of heavy vehicles (class 7-8): Simulation and benchmarking. SAE Paper 2000-01-2209.

Patankar, S., Spalding, D., 1972. A calculation procedure for heat, mass and momentum transfer in threedimensional parabolic flows. Int. J. Heat Mass Transfer 15, 1787-1806.

Perry, A. E., Chong, M. S., 1987. A description of eddying motions and flow patterns using critical-point concepts. Ann. Rev. Fluid Mech. 19, 125 - 155.

Pope, S. B., 2000. Turbulent Flows, 1st Edition. Cambridge University Press, Cambridge. 
Smagorinsky, J., 1963. General circulation experiments with the primitive equations. Monthly Weather Review 91 (3), 99-165.

Sujudi, D., Haimes, R., 1995. Identification of swirling flow in 3-d vector fields. AIAA Paper 95-1715. 